\title{
ORIGINAL
}

\section{MORTALIDAD INNECESARIAMENTE PREMATURA Y SANITARIAMENTE EVITABLE EN COSTA RICA}

\section{Fernando Llorca Castro (1) y Vicente Ortún Rubio (2)}

(1) Fundación ACTIVA Internacional.

(2) Departamento Economía y Empresa y Director del Centro de Investigación en Economía y Salud, Universidad Pompeu Fabra, Barcelona.

\section{RESUMEN}

Fundamentos: Para desarrollar políticas y estrategias orientadas a mitigarlas es fundamental identificar las desigualdades. El objetivo del trabajo es analizar las variaciones de la Mortalidad Innecesariamente Prematura y Sanitariamente Evitable (MIPSE) para cada uno de los 81 cantones de Costa Rica durante el período 2000-2005.

Métodos: Se aplicó la clasificación MIPSE propuesta por miembros del Servicio de Información y Estudios de la Dirección General de Recursos Sanitarios de Catalunya. Mediante el empleo de el Indicador de Desarrollo Socioeconómico (IDSE) establecido por economistas de la Universidad de Costa Rica, previa estandarización de la población, se ordenaron los cantones en grupos por quintiles (I el más rico, $\mathrm{V}$ el más pobre).

Resultados: Se encontraron como causas principales de mortalidad MIPSE la enfermedad isquémica del corazón (19,55\% causas MIPSE), accidentes de tránsito con vehículos a motor (11,60\%), enfermedades cerebrovasculares $(6,95 \%)$, perinatal $(6,92 \%)$ y suicidios $(5,14 \%)$. Conclusión: La mortalidad por HIV y el Sida, el cáncer de mamá en mujeres, cáncer de cuerpo de útero, cáncer de piel y por hepatitis secundaria al consumo de alcohol, afectan más a los cantones con mayores ingresos. La mortalidad por hiperplasia benigna de próstata, la materna asociada al embarazo, parto o puerperio y la hernia abdominal afectan más a los de menor nivel económico. Se identificaron dos grupos de MIPSE con desigualdad equidistribuida: leucemia y enfermedades cardiovasculares congénitas.

Palabras clave: Desigualdades en la salud. Evaluación de resultado (atención de salud). Mortalidad. Costa Rica.

\section{ABSTRACT \\ Unnecessary premature and avoidable mortality in Costa Rica}

Backgrounds: With the intention of establishing economic inequities, the article analyzes the variations of the Unnecessarily Premature and Sanitarily Avoidable Mortality (MIPSE) of each of the 81 cantons of Costa Rica during 2000-2005. It is important to identify those inequities, to establish policies and strategies trying to mitigate them.

Method: It applies the MIPSE classification proposed by members of the Information and Studies Service, of the Catalunya's Sanitary Resources Headquarter, Spain. By an Indicator of Socioeconomic Development (IDSE) of a University of Costa Rica economist's team, it organised each canton in groups of quintiles (I for the richest, $\mathrm{V}$ for the poorest), previous people standardization.

Results: We found as a major causes of mortality MIPSE in the country: Heart Isquemic Disease (19,55\% MIPSE causes), Traffic Accidents with Motor Vehicles $(11,60 \%)$, Brain Vascular Disease $(6,95 \%)$, Perinatal $(6,92 \%)$ and Suicide $(5,14 \%)$

Conclusion: The VIH infection - AIDS mortality, the Best Cancer in Women, Uterus Cancer, Skin Cancer and Hepatic Disease Secondary to Alcohol Consumption, affects more the cantons with better financial conditions and the Prostate Benign Hyperplasia mortality, Mothers mortality related with Pregnancy, Childbirth or Puerperal Stage and the Abdominal Hernia mortality, affects more to those with worst economic level. Two MIPSE groups were identified with similar inequality: Leukaemia and Congenital Cardiovascular Disease.

Key words: Inequalities. Evaluations. Mortality. Costa Rica.

Correspondencia:

Fernando Llorca Castro

Fundación ACTIVA Internacional

fllorca@activainternacional.org

Apartado Postal 200

28080, Madrid 


\section{INTRODUCCIÓN}

Costa Rica, el país más feliz del mundo ${ }^{1}$, destaca entre sus vecinos por sus buenos indicadores de desarrollo humano. La supresión del ejército en 1949 y la consolidación de las garantías sociales e individuales de 1943, permitieron una política redistributiva que ha facilitado el acceso a la educación y la sanidad, una esperanza de vida general al nacer de más de 79 años y una tasa de mortalidad infantil de 9,77 por mil para el 2005 (Indicadores Generales de Costa Rica, Centro Centroamericano de Población).

La relación existente entre la mortalidad y la inequidad se ha establecido desde hace tiempo ${ }^{2}$. Muchas de estas investigaciones refuerzan la idea de utilizar la mortalidad como indicador de desempeño de los sistemas de salud de una región, zona o nación y para detectar desigualdades. Los esfuerzos para eliminarlas han sido insuficientes, inclusive para las sociedades que han avanzado más en este terreno ${ }^{3}$. Costa Rica no parece ser la excepción. A pesar de los logros y las mejoras en accesibilidad a los servicios de salud y la universalidad de las políticas de salud públicas que presenta el país ${ }^{4,5}$, persisten importantes desigualdades en salud aún sin identificar.

Muchas veces se ha asociado la mortalidad con la capacidad que tienen los sistemas de salud de mitigarla ${ }^{6}$. Un buen sistema de salud, a pesar de los cambios políticos que experimente ${ }^{7}$, debe ser universal y no establecer diferencias en su accesibilidad desde la zona rural o la urbana $^{8}$, por la edad o el sexo de las personas aseguradas ${ }^{9,10}$ o por las mejoras terapéuticas que la tecnología va incorporando ${ }^{11}$. Sabemos que las corrientes políticas influyen sobre los indicadores de salud $^{12}$ y que, por lo tanto, estos indicadores de mortalidad los debemos ir depurando ${ }^{13}$.

Como se mencionó, la relación entre el nivel socioeconómico y la enfermedad es sólido ${ }^{14}$. Otros de sus asociados son el nivel de educación ${ }^{15}$, el estilo de vida ${ }^{16}$, las desigualdades en sí mismas ${ }^{17}$, el desempleo como tal ${ }^{18}$, el matrimonio o el estrés ${ }^{19} \mathrm{u}$ otras causas psicosociales ${ }^{20}$ así como uno de los fenómenos más documentados, la raza o la inmigración ${ }^{21}$. En Costa Rica los estudios que se han realizado se han limitado a identificar las causas de muerte más frecuentes.

La mortalidad innecesariamente prematura y sanitariamente evitable (MIPSE) permite aproximar, por un lado, la efectividad de servicios sanitarios y políticas de salud. Por otro, cuando se analizan geográficamente y según renta las causas MIPSE ${ }^{22}$ como se hace en este artículo, conseguimos una buena imagen del grado de equidad conseguido.

El objetivo de este trabajo es analizar las variaciones de la MIPSE en los 81 cantones de Costa Rica durante el período 2000-2005, identificando inequidades relacionadas con el nivel medio de ingreso económico por cantón.

\section{MATERIAL Y MÉTODOS}

Se analizaron la población universal y la mortalidad absoluta. Se juntaron las causas de MIPSE en 34 grupos, al no existir una propia para Costa Rica, utilizando los códigos de la Clasificación Internacional de Enfermedades en su versión 10 (CIE 10). De ellos, 27 se consideraron como causas vulnerables de intervenciones de los servicios de atención sanitaria (ISAS) y 7 como causas sensibles a intervenciones de políticas de salud intersectoriales (IPSI). Como indicador de desarrollo socioeconómico (IDSE) se empleó el Ingreso Medio por Hogar y por Cantón que desarrollaron economistas de la $\mathrm{UCR}^{23}$ en 2005. Mediante ese IDSE de Hogar por Cantón en orden al totalizarlos se obtuvieron quintiles para cada grupo de MIPSE y con subtotales por ISAS e IPSI.

Partiendo de la población bruta que arroja el mismo sistema del CCP en un solo bloque 
entre los años 2000 al 2005, una de las bases de datos más fiables del país, se obtuvo la tasa bruta y se ajustó empleando la tasa estándar por edad mundial (TEE Mundial), obteniendo finalmente las tasas ajustadas por edad (TAE Mundial) y sus respectivos quintiles para cada MIPSE y subtotales ISAS e IPSI. Finalmente, se comparó cada quintil identificando los casos de sobremortalidad a favor de ricos y pobres y los casos de mortalidad equidistribuida aplicando la siguiente fórmula:

Tasa ajustada por edad (TAE): TAE $_{\mathrm{i}}=$ $100.000 * \sum m_{\mathrm{i}} / n_{\mathrm{i}} * w_{\mathrm{i}}$

$$
\begin{aligned}
& \mathrm{i}=\text { Cada Grupo de MIPSE } \\
& m=\text { MIPSE } \\
& n=\text { Población } \\
& w=\text { TEE }
\end{aligned}
$$

Además, para cada MIPSE TAE Mundial, se obtuvo una Ratio de cada Grupo $\left(\mathrm{R}_{\mathrm{i}}\right)$ quintiles I y V (Ratio QI $\left./ \mathrm{QV}_{\mathrm{i}}\right)$ :

$$
\begin{aligned}
& \mathrm{R}_{\mathrm{i}}=\mathrm{QI}_{\mathrm{i}} / \mathrm{QV}_{\mathrm{i}} \\
& \mathrm{i}=\text { Cada Grupo de MIPSE } \\
& \mathrm{QI}=\text { Quintil I } \\
& \mathrm{QV}=\text { Quintil V }
\end{aligned}
$$

El coeficiente de correlación de Pearseon (CCPe):

$$
\begin{aligned}
& \mathrm{CCP}_{\mathrm{i}}=\Sigma x_{\mathrm{i}}: \Sigma y_{\mathrm{i}} \\
& \mathrm{i}=\text { Cada Grupo de MIPSE } \\
& x=\mathrm{TAE} \\
& y=\mathrm{IDSE}
\end{aligned}
$$

Los intervalos de confianza de las Ratio QI / QV de cada MIPSE TAE Mundial se utilizó la siguiente fórmula:

$$
\begin{aligned}
& \exp \left[\ln (\mathrm{TAE} \mathrm{QI} / \mathrm{TAE} \mathrm{QV}) \pm 1,96 \mathrm{v} 1 / M_{i}\right. \\
& \left.+1 / M_{N}\right] \\
& \exp =\text { el exponencial } \\
& \ln =\text { logaritmo natural } \\
& M_{i}=\text { MIPSES de cada Grupo } \\
& M_{N}=\text { MIPSES del país }
\end{aligned}
$$

\section{RESULTADOS}

En la tabla 1 se resumen los resultados explícitos obtenidos para cada causa MIPSE y los grupos subtotales específicos de ISAS e IPSI.

Descripción y análisis de resultados totales. Las MIPSE representan en torno al $60,4 \%$ de la mortalidad total. Las 5 causas MIPSE más frecuentes en Costa Rica para 2000-2005 representan el 50,16\%. La MIPSE 16 o de Enfermedad Isquémica del Corazón con un 19,55\%, la MIPSE 31 de Accidentes de Tránsito con Vehículos a Motor con $11,60 \%$, la 12 o de Enfermedades Cerebrovasculares con $6,95 \%$, la 14 de Causas perinatales $6,92 \%$ y finalmente, la MIPSE 32 de Suicidios con un 5,14\%.

La mortalidad bruta total MIPSE durante el 2000 y el 2005 por Cantón en Costa Rica se ilustra en la figura 1, en la que se observa que la mortalidad bruta se concentra en la Gran Área Metropolitana.

El coeficiente de correlación de Pearseon $(\mathrm{CCPe})$ mostró una relación lineal débil ya que fue cercano a 12 veces y 17 oportunidades negativo, entre el ingreso y la TAE Mundo. Por otro lado, el intervalo de confianza (IC95\%) fue estadísticamente significativo para 26 de los casos, incluidos todos lo que descritos y analizados a continuación.

Descripción y análisis de resultados subtotales ISAS e IPSI. En la tabla 1 se aprecia cómo la mortalidad que depende de intervenciones de los sistemas de atención sanitaria (ISAS) y que explica un 58,19\%, mantuvo una tendencia a la baja en favor de los quintiles de cantones con menos ingresos económicos. Sin embargo, la ratio QI/QV ISAS fue levemente superior a 1, mostrando que la tendencia a favor de los de menor ingreso era leve.

Respecto a la mortalidad que se podría ver influenciada por intervenciones de políticas 


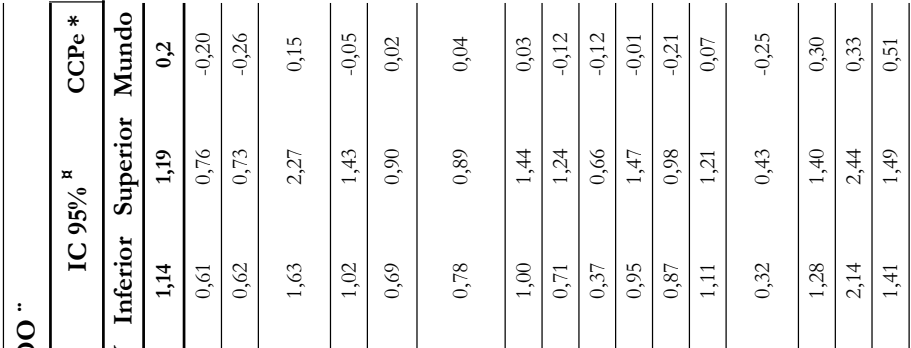

$\sum_{\text {䆟 }}^{\text {号 }}$

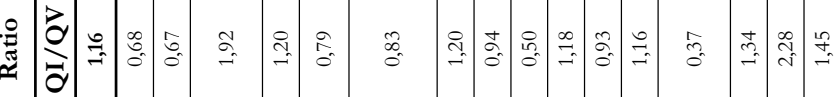

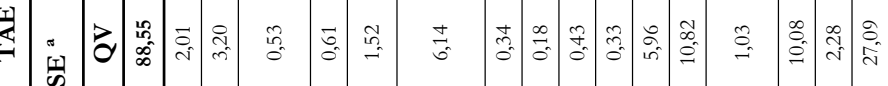

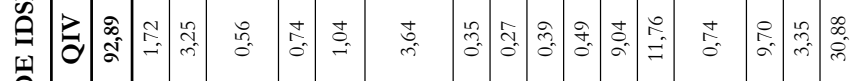
का 。 व

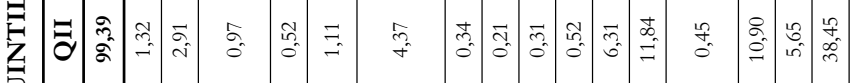

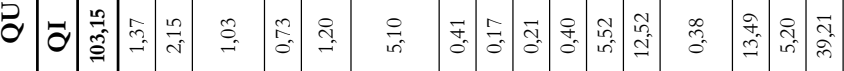

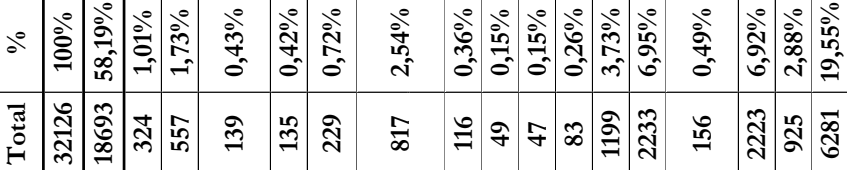
ํํำ 茪 능 政 竎

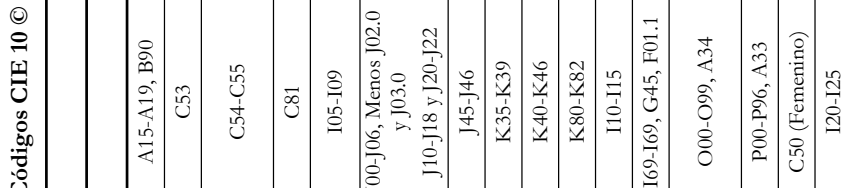

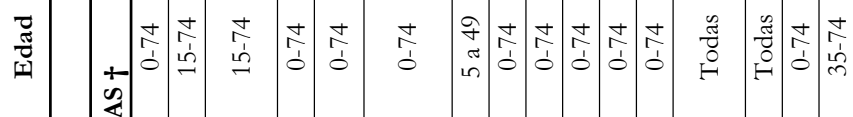
缹 吾

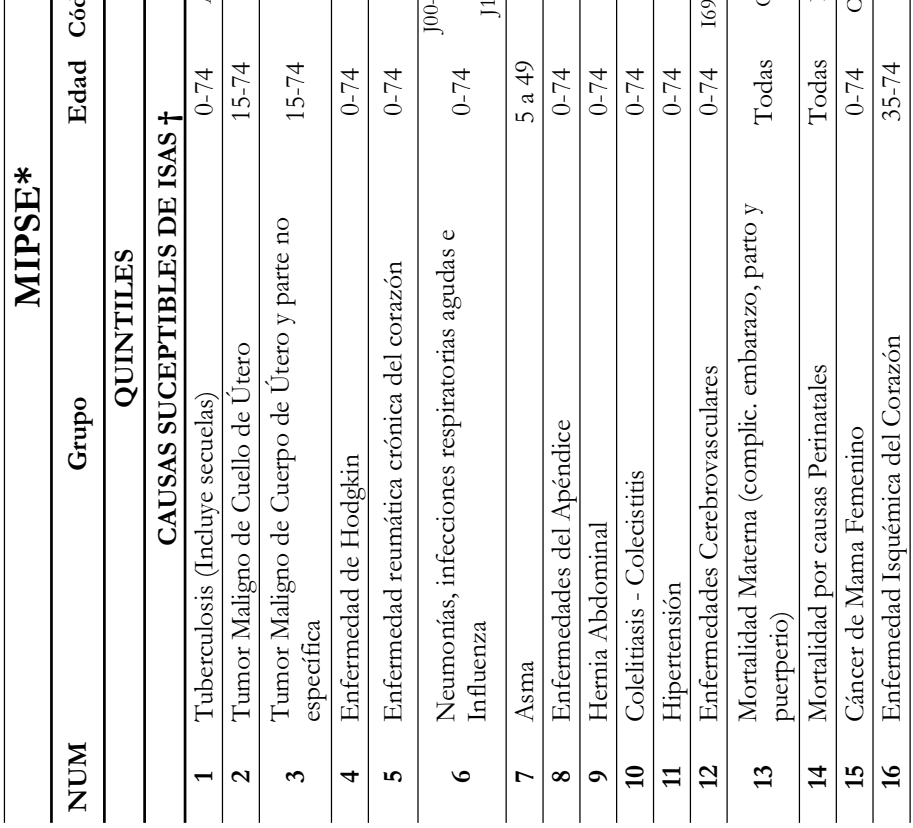




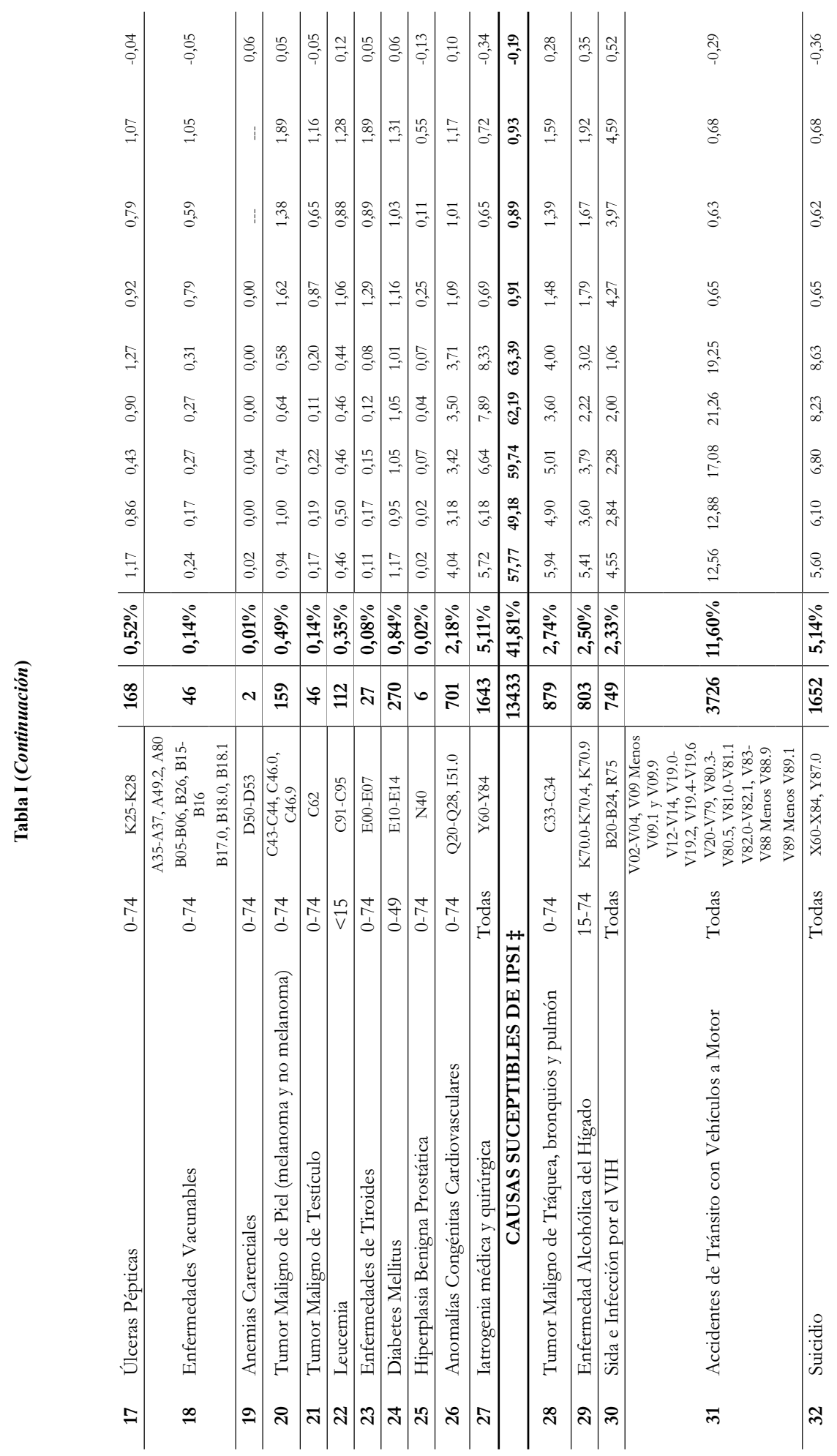




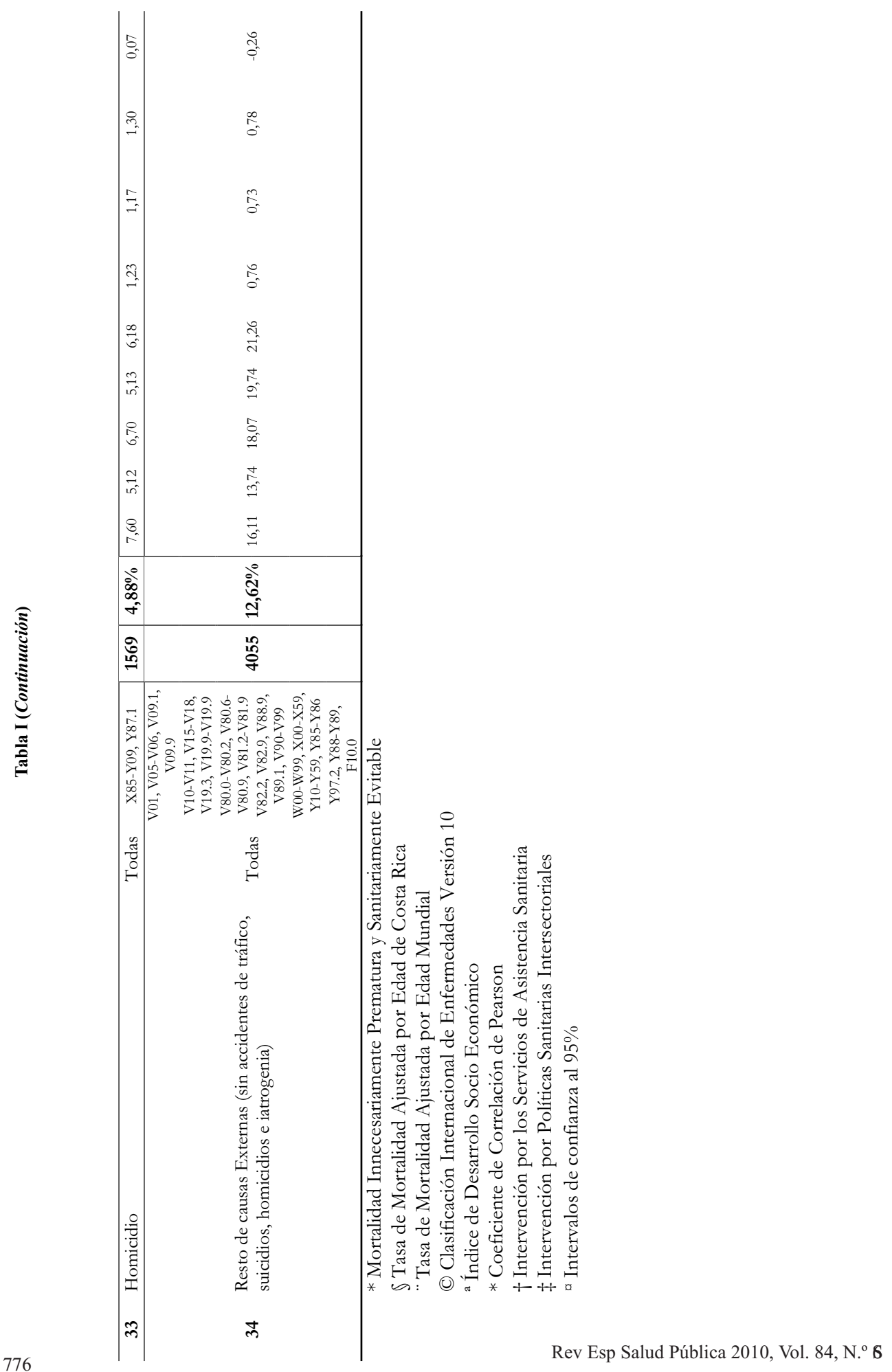


Figura 1

Gran total de mortalidad innecesariamente prematura y sanitariamente evitable para ambos sexos por cantones de Costa Rica 2000-2005

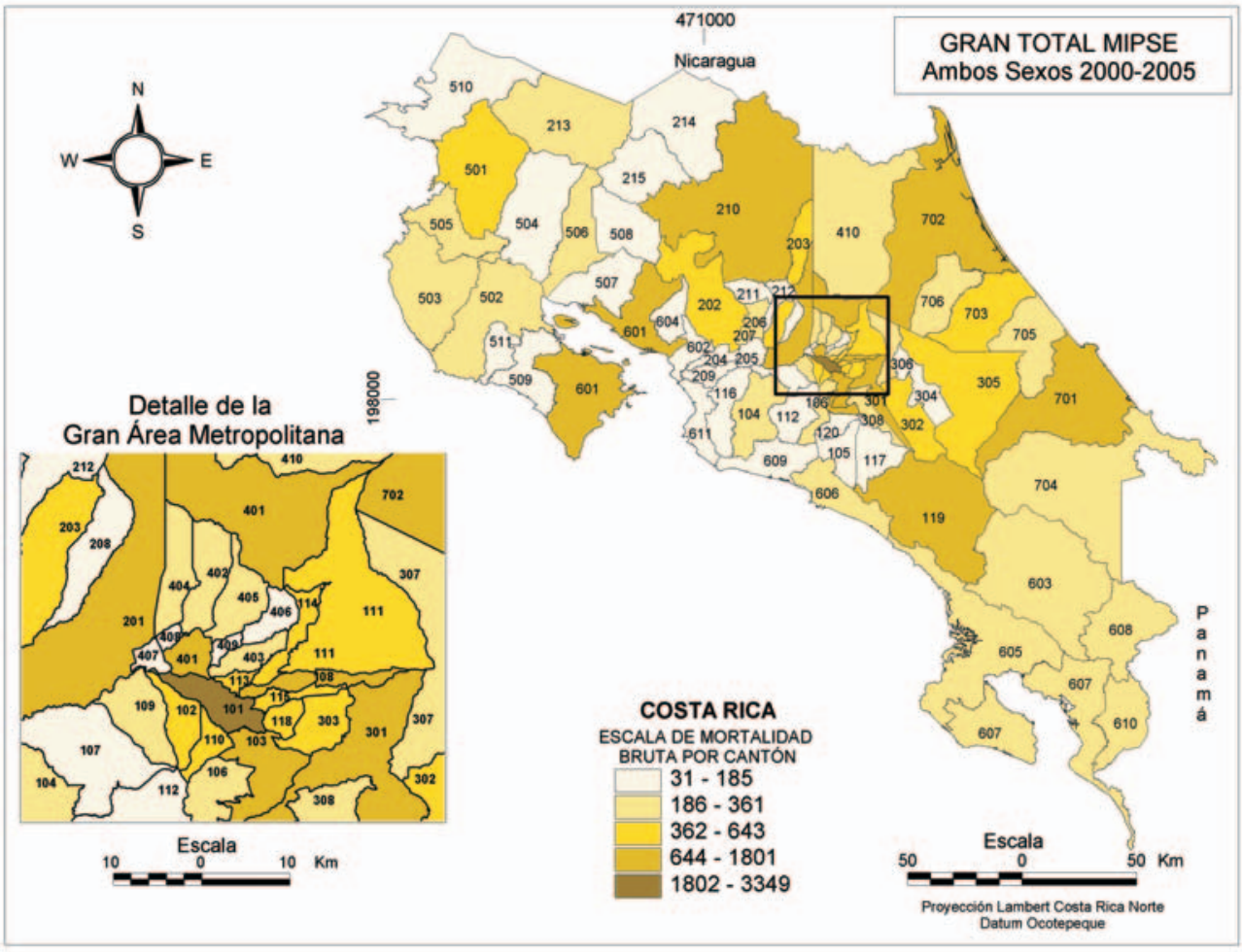

de salud intersectoriales (IPSI) y que ilustraba el $41,81 \%$ de los fallecimientos MIPSE, se apreció una leve inclinación a favor de los cantones de medio-mayor ingreso, sin estar clara o definida, y que al ser alta en los extremos mostraba una Ratio QI / QV IPSI menor a 1 .

El gran total de MIPSE ISAS en Costa Rica 2000-2005 se puede observar por cantón en la figura 2. Comparándolo con el gran total de mortalidad para ambos sexos (figura 1) se detallaron pocas diferencias salvo para los casos de alta concentración de mortalidad en el Cantón de Alajuela o 201, el Cantón de Desamparados o 103 y disminución en MIPSE para los cantones rurales de Osa o 605, Pococí o 702 y Siquirres o 703.

El gran total de MIPSE IPSI en Costa Rica 2000-2005 se puede observar por cantón en la figura 3. En él se ilustran diferencias con la figura 1 de aumento de MIPSE exclusivamente, para los cantones de Aserrí o 106, Sarapiquí o 410, Nicoya o 502, Santa Cruz o 503, Talamanca o 704, Matina o 705, y Guácimo o 706.

Descripción y análisis de resultados con sobremortalidad. Relacionado a cada grupo de MIPSE se pudo demarcar que en Costa Rica para los años analizados persistían 
Figura 2

Gran Total de Mortalidad Innecesariamente Prematura y Sanitariamente Evitable para Intervenciones de los Servicios de Atención Sanitaria por cantones de Costa Rica 2000-2005

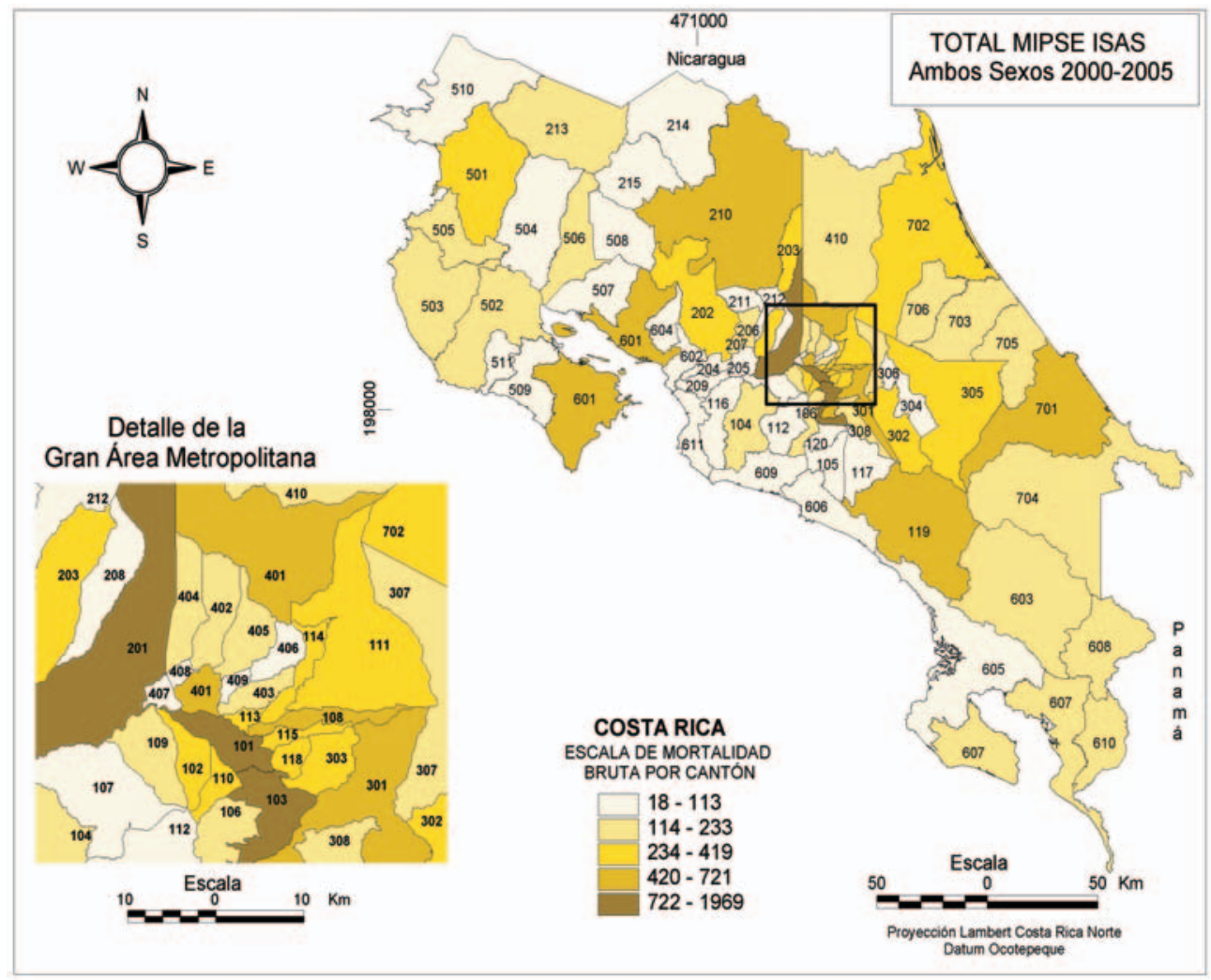

desigualdades respecto a la tasa de mortalidad estadísticamente significativas, muy importante entre los quintiles más ricos $(\mathrm{QI})$ y los más pobres (QV) en algunas patologías, como el HIV positivo y el SIDA, donde dicha mortalidad era considerablemente más alta que la encontrada entre los pobres (figura 4), con un porcentaje de MIPSE de 2,33\%, al igual que la enfermedad alcohólica del hígado, con un porcentaje de participación de la mortalidad total de 2,50\%.

Se repetía el fenómeno en patologías como el cáncer de mama femenino con un $2,88 \%$ de los fallecimientos, para su quintil I y sobre todo para su quintil II. Casos como los del tumor maligno uterino con un porcentaje de MIPSE de $0,43 \%$ del total y el tumor maligno de piel con un $0,49 \%$, mantenían un comportamiento muy similar al del cáncer de mamá.

La sobremortalidad que perjudica a los pobres se encontraba en primer lugar en la hiperplasia benigna de la próstata (figura 5 ), cuya mortalidad fue 5 veces mayor en los cantones pobres que en los ricos, pasando por un pico igualmente importante en los cantones de ingreso medio o quintil III. 
Figura 3

Gran Total de Mortalidad Innecesariamente Prematura y Sanitariamente Evitable para Intervenciones de Políticas de Salud Intersectoriales por cantones de Costa Rica para el período 2000-2005

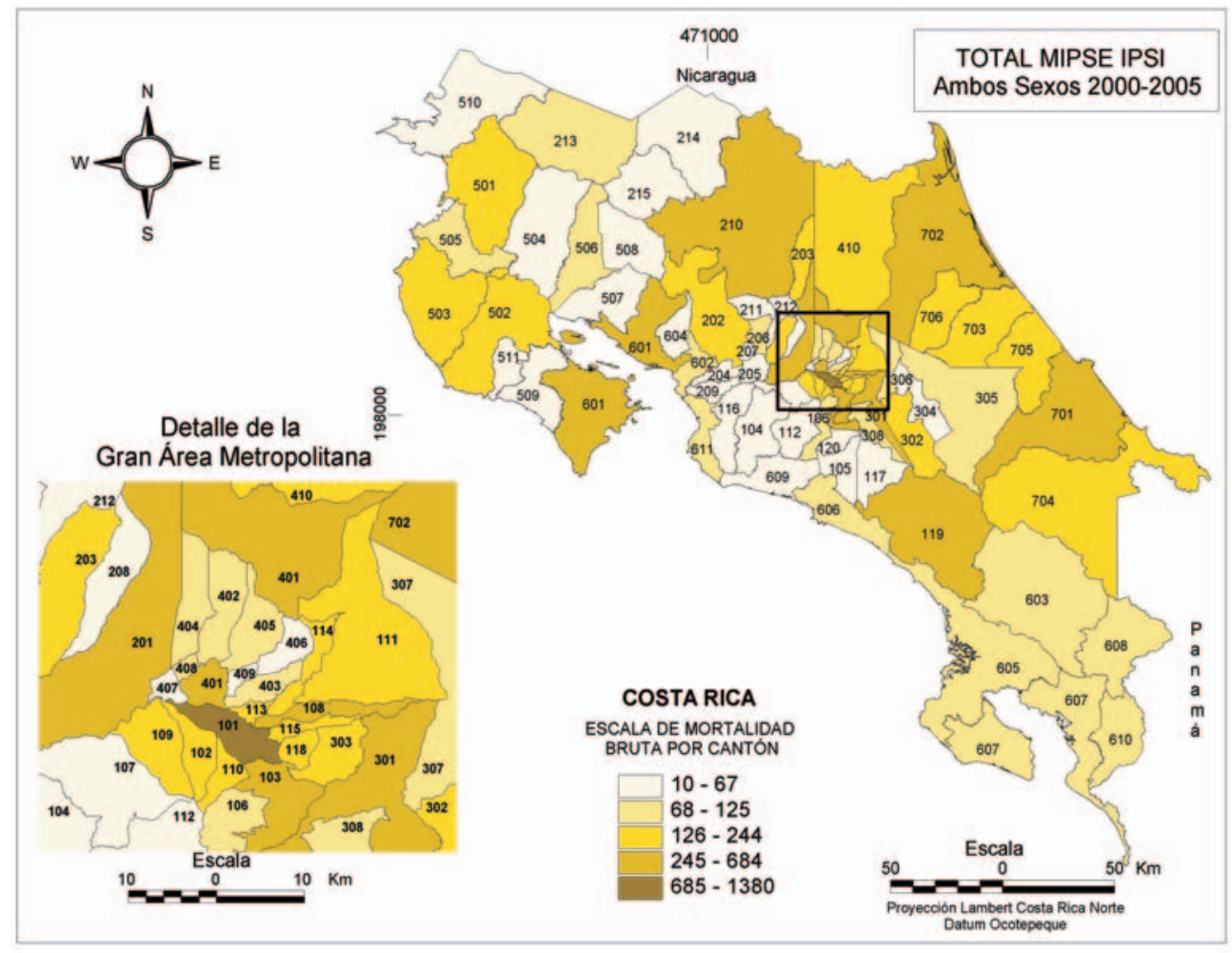

Fuente: Elaboración propia.

La mortalidad materna como complicación del embarazo, parto o puerperio significó un $0,49 \%$ del total de defunciones durante los años 2000 y 2005. El comportamiento en la figura 5 es escalonado y uniforme en aumento de la misma en perjuicio de los más pobres.

Finalmente, para la sobremortalidad que afectaba a los más desposeídos estaban los problemas de hernia abdominal, que afectaron a un $0,15 \%$ de los fallecidos, variando alternamente su comportamiento entre los quintiles.

Descripción y análisis de resultados con mortalidad equidistribuida. Dentro del resto de grupos de MIPSE analizados entre los años 2000 y 2005 en Costa Rica, destacaron dos patologías que se han comportado especialmente igualitarias a la hora de asignar infortunios a todos los cantones del país por igual. Tenemos inicialmente las MIPSE causadas por leucemias (figura 6) con un nivel de mortalidad muy similar en todos los quintiles, un aumento puntual del quintil II y una porcentaje general de mortalidad del $0,35 \%$. La segunda patología detectada fueron las anomalías congénitas cardiovasculares, con un aporte a la mortalidad global de ignorar de un $2,88 \%$. 
Figura 4

Quintiles de Mortalidad Innecesariamente Prematura y Sanitariamente Evitable con Sobre mortalidad del Quintil I o de Ricos en Costa Rica para el período 2000 - 2005, en Tasa Ajustada por Edad Mundial

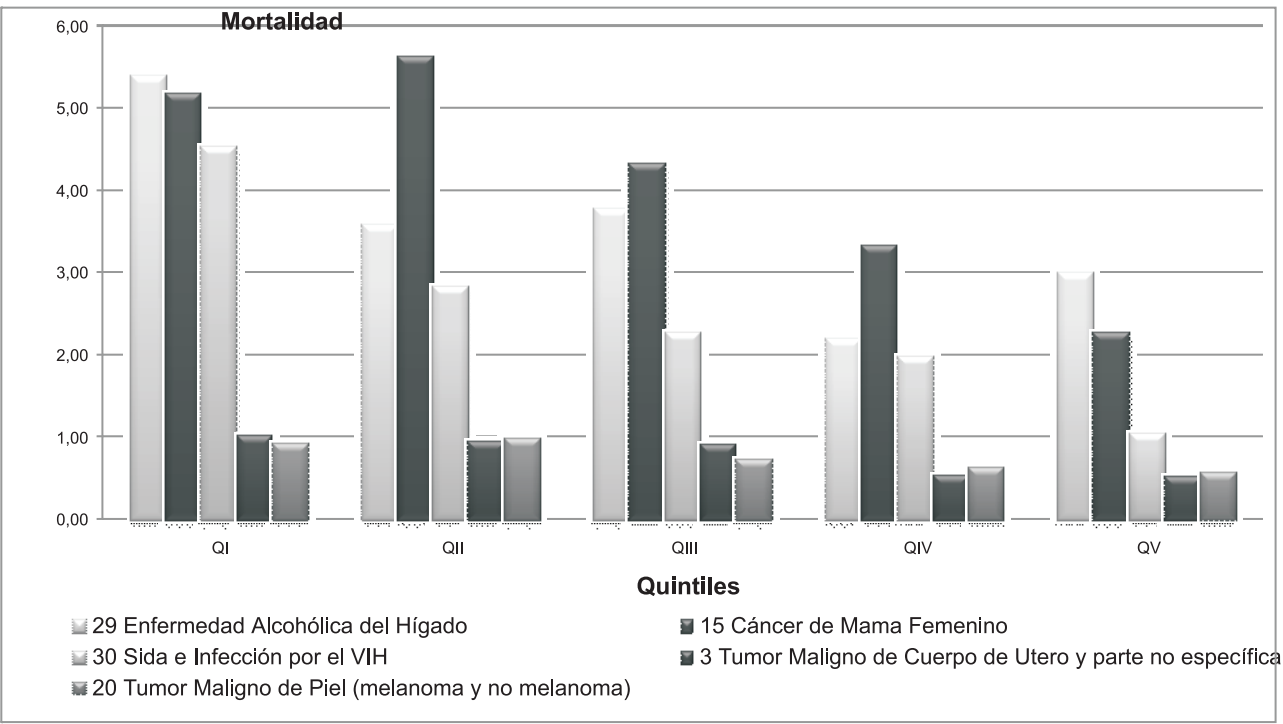

Figura 5

Quintiles de Mortalidad Innecesariamente Prematura y Sanitariamente Evitable con Sobre mortalidad del Quintil V o de Pobres en Costa Rica para el período 2000 - 2005, en Tasa Ajustada por Edad Mundial

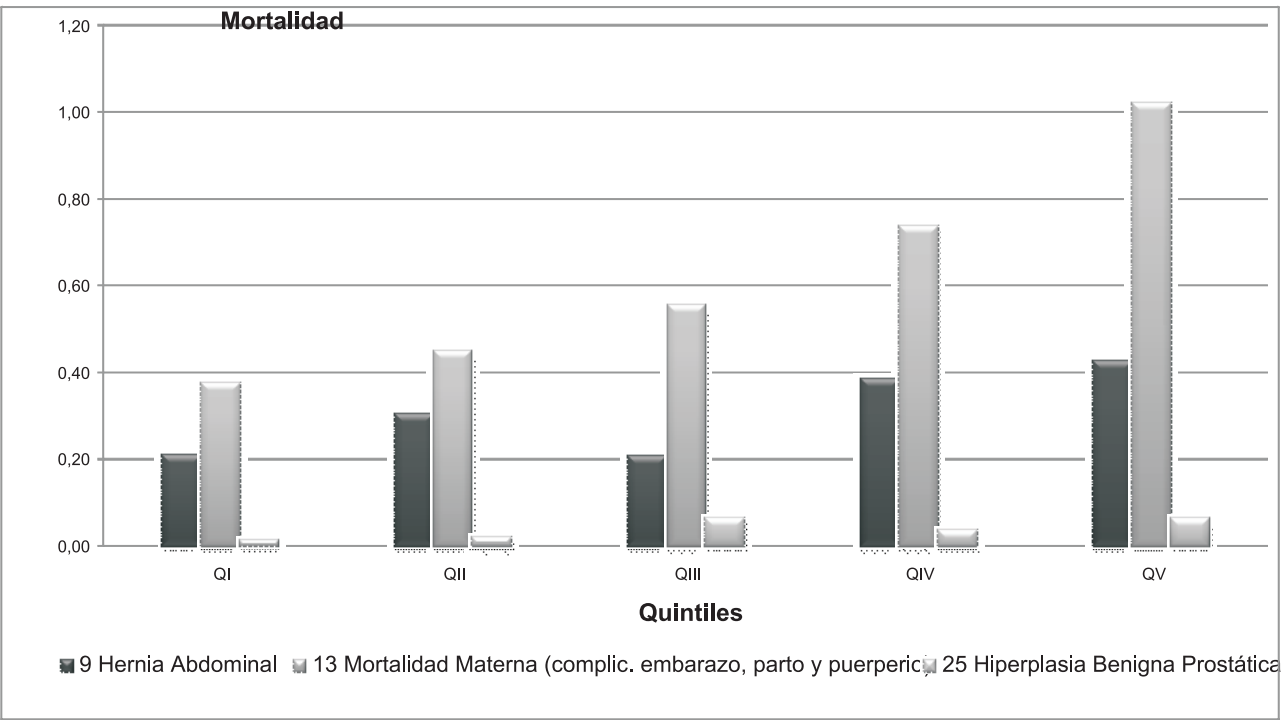


Figura 6

Quintiles de Mortalidad Innecesariamente Prematura y Sanitariamente Evitable Equidistribuida en Costa Rica para el período 2000 - 2005, en Tasa Ajustada por Edad Mundial

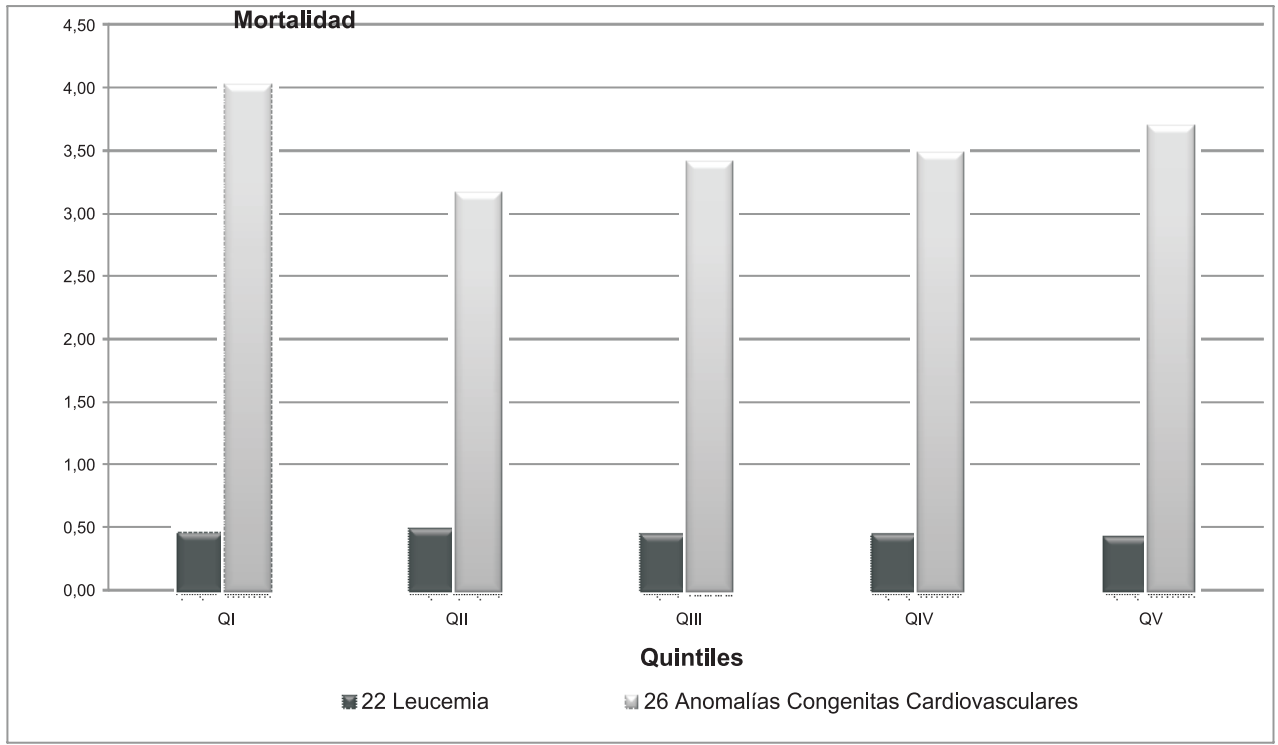

\section{DISCUSIÓN}

La muerte innecesariamente prematura y sanitariamente evitable se presenta en Costa Rica con diferencias sutiles y explicables entre los grupos de intervenciones ISAS e IPSI. Las primeras repercuten negativamente en cantones cercanos a la capital, que suelen ser de mejor nivel educativo o socioeconómico y positivamente sobre unos pocos cantones rurales alejados de la capital, en los cuales se mantiene con un menor nivel de riqueza y educación.

En cambio, en la gran área metropolitana, sucede todo lo contrario que en la observación anterior. Son los cantones de mayor cercanía a la capital, con mejor nivel educativo, mejor nivel de ingreso económico por familia, de poblaciones urbano o urbano marginales, los que no se ven afectados. No así, los niveles de MIPSE en las zonas rurales y urbanas marginales, donde para las
IPSI, se muestra que aumentan las diferencias.

Como principales limitaciones del estudio podemos señalar las que se derivan de los datos utilizados. Partimos del hecho de que el registro de las causas de cada muerte del CCP son los oficiales del país. La clasificación MIPSE catalana es muy similar a otras empleadas en todo el mundo para estos casos y Costa Rica no posee una propia. Los grupos del CIE 10 utilizados para la lista son los que se manejan en el país y la materia prima del IDSE de la UCR son también datos oficiales. Ante el volumen de información manejada, y gracias a que el estudio fue amplio en analizar todas las causas MIPSE, se omitió analizarlo por sexo, edad, etnia, etc.

Otra limitación es que el período de tiempo empleado de 5 años comprendido entre los años 2000 y 2005 depende del censo de la 
población costarricense que se llevó a cabo en el año 2000 y de estimaciones basados en ella para los años siguientes.

En Costa Rica existen dudas razonables sobre la calidad de los registros de las defunciones, basados en lo que el médico anota, inspiradas en criterios clínicos a la hora de certificar la muerte y en lo encontrado en los hallazgos objetivos de la autopsia. De hecho, se encontró un 44,8\% de discrepancia de 173 casos para el año 2000 entre ambas ${ }^{24}$.

Sobre las principales causas de muerte en Costa Rica entre los años 2000 y 2005 se puede decir que son también muy importantes en el resto del mundo. La primera de ellas, la enfermedad isquémica del corazón, ha sido estudiada ${ }^{25}$ y se ha documentado la relación que tienen directamente sus factores de riesgo y la mortalidad en sí, manteniendo inequidades ${ }^{26}$. En Costa Rica se ha analizado la mortalidad por esta causa, encontrando un comportamiento similar al de otros países más desarrollados ${ }^{27}$, con tendencias a la baja sin descuidar algunos factores de riesgo que aumentan ${ }^{28}$.

Del resto de causas en orden de frecuencia sobresale la MIPSE 31 de accidentes de tránsito con vehículo a motor que también es de importancia en el resto del mundo como se ha documentado en Europa ${ }^{29}$ y en América incluyendo a Costa Rica ${ }^{30}$, que no solamente no es la excepción sino que la mortalidad en este apartado es preocupantemente alta y paralela al aumento del desarrollo económi$\mathrm{co}^{31}$ al igual que en otros rincones de las Américas, por lo que se considera un problema urgente de Salud Pública ${ }^{32}$, superando a otros grupos de MIPSE como al MIPSE 12 de Enfermedades Cerebrovasculares o el MIPSE 14 de causas perinatales. Esta última, está muy relacionada con el nivel socioeconómico de los pueblos como ya habíamos señalado ${ }^{33}$ y en el caso de Costa Rica se mantiene como una causa importante ya que es una nación en vías de desarrollo $^{34}$. Sobre la MIPSE 32 o de suicidios es también una causa frecuente en países desarrollados $^{35}$ como sucede en Costa Rica ${ }^{36}$.

Las enfermedades que afectan más a los cantones con mayor ingreso por familia son causas de fallecimiento usualmente encontradas en países desarrollados, salvo para el caso del HIV o el Sida, que es la causa que afecta con mayor desigualdad a los más enriquecidos en Costa Rica, no así en el resto del mundo, donde en países desarrollados se ha logrado controlar la mortalidad y en países más pobres hace estragos a sus anchas ${ }^{37}$. En Costa Rica se han sugerido problemas a la hora de notificar la condición diagnóstica en las zonas costeras que casualmente son las más pobres, sin embargo el aumento de HIV continúa y la mortalidad por Sida parece disminuir $^{38}$, probablemente asociado a la utilización de terapia antirretroviral, por lo que ha disminuido la mortalidad intrahospitalaria. Sin embargo el grupo más afectado es el de los hombres jóvenes y solteros o de zonas rurales ${ }^{39}$.

Respecto a la mortalidad causada por cáncer en general ya se ha asociado con un nivel alto de ingreso económico ${ }^{40}$. También se ha asociado a patologías nutricionales, como la obesidad, en el caso de neoplasias del tubo digestivo $^{41}$ o directamente asociada al tumor maligno de mama o de útero ${ }^{42}$, que en este caso sí son unas de las afecciones que inclinan la balanza mortal en detrimento de los que más poseen.

La MIPSE 15 de cáncer de mama femenino se relaciona con la adecuada aplicación del auto-examen de mama y con el acceso a la atención primaria ${ }^{43}$. No obstante, esto no explica por qué razón las mujeres que viven en los cantones más ricos se vean más afectadas. Sin embargo, en algunos de los cantones Gran Área Metropolitana del país se han implementado recientemente nuevos modelos de atención primaria públicos, gestionados por entidades privadas, cooperativas $u$ organizaciones sin fines de lucro. A pesar de las críticas en la forma de implementación 
del cambio ${ }^{44}$, se ha demostrado cuantitativamente su mejor desempeño en los años noventa y el nuevo siglo ${ }^{45}$.

Durante años no ha existido una buena cobertura por parte de la Seguridad Social de esta población adinerada, ya que no utilizaba los servicios y controles estatales. En los sitios más ricos, las personas mantenían su vigilancia de enfermedades crónicas o de medicina preventiva en general por su cuenta. Algunas veces con controles privados muy completos y otras sin control sistematizado. Aunado a esto, en un importante y reciente estudio, directamente se ha demostrado un patrón genético de herencia en 23 familias costarricenses que consultaban en el Hospital San Juan de Dios en la ciudad Capital, San José ${ }^{46}$.

Después del cáncer de mama femenino hay patologías pertenecientes a este grupo que la acompañan afectando a la población mejor posicionada económicamente del país. Hablamos puntualmente de la MIPSE 3 o del tumor del cuerpo del útero y otros y la MIPSE 20 o tumor maligno de piel. Para el primer caso, junto con el cáncer de mama femenino, se ha asociado a la utilización de terapia hormonal sustitutiva en las mujeres durante la menopausia ${ }^{47} \mathrm{y}$ definitivamente este tipo de tratamientos estuvo inicialmente al alcance de personas económicamente solventes. En todo caso, el diagnóstico temprano como suele ser común para las patologías neoplásicas en general, es fundamental y para el proceso diagnóstico de los tumores del cuerpo del útero se requiere una atención del segundo nivel o especializada.

El cáncer de piel es bien conocido por su relación con la exposición inadecuada a los rayos ultravioleta ${ }^{48}$, ya que se ha asociado a algunos factores de riesgo como tener la piel blanca, la exposición prolongada a las radiaciones, la moda de estar bronceado, o la combinación con el uso de medicamentos que aumentan la fotosensibilidad, entre otros. Encontrando grupos de clases social que puedan estar expuestos a esos factores de riesgo, se podría explicar la desigualdad en la mortalidad en contra de los más potentados financieramente.

En Costa Rica se ha documentado con claridad $^{49}$ que existen conductas sociales que aumentan la exposición de la piel a los factores de riesgo, como pueden ser la práctica de deportes de playa o acuáticos, sobre-exponerse a las máquinas bronceadoras artificiales y combinar la exposición al sol con la ingesta simultánea de medicamentos. Si a esas razones le agregamos el hecho de que vacacionar con frecuencia en la playa sin tomar las medidas apropiadas y aunado a que se ha demostrado que la mayor cantidad de rayos ultravioleta se reciben de niño o en zonas como el rostro $(90 \%$ de pre-malignidad), podríamos pensar que estas personas tienen mayor peligro. En todo caso, en Costa Rica y en otros lugares se ha demostrado que las personas que utilizan protector solar con frecuencia lo emplean mal, en poca cantidad, mal distribuido y, confiados, se exponen durante más tiempo al sol que las personas que no los utilizan siempre.

Sobre la MIPSE 29 de la enfermedad alcohólica del hígado y su inclinación en contra de los menos necesitados económicamente, no podríamos sugerir ninguna causa específica y, quizás en parte, se deba a que no existen suficientes estudios sobre el problema y verdadero impacto en las clases sociales más altas. Se encontró que las profesiones más frecuentemente asociadas a los fallecimientos por consumo de etanol son la construcción y el mantenimiento, la agricultura y el comercio, con un perfil de personas solas y bajo nivel de educación ${ }^{50}$.

Poco sorprende que la mortalidad encontrada en los cantones más pobres incluya en primer lugar a la MIPSE 25 o de hiperplasia benigna de próstata, muy asociada a un grupo de edad vulnerable de la población, el adulto mayor masculino. Las limitaciones económicas y de acceso a los servicios sani- 
tarios son especialmente notorias para este colectivo.

La mortalidad materna como consecuencia del embarazo, parto o puerperio o MIPSE 13, afecta especialmente a las mujeres de escasos recursos económicos ${ }^{51}$, no solo porque la cantidad de embarazos por mujer suele ser mayor sino porque algunos de ellos se producen en mujeres adolescentes y en mujeres mayores de 40 años. Esta última se da por dos causas, por retraso de la gestación intencionalmente y mujeres con muchos embarazos, cuya condición económica suele ser más bien baja y de las zonas rurales.

Finalmente, dentro de las patologías que afectan más a los menos agraciados, tenemos la MIPSE 9 o por hernia abdominal, de la cual solamente se puede señalar que se ha estudiado muy poco y no encontramos más justificación que las posibles barreras de acceso típicas de estos casos.

Dentro de los datos más interesantes se encuentran dos grupos de MIPSE cuya distribución entre los distintos quintiles de los cantones ordenados en función del nivel de ingreso por familia es muy uniforme. El primero de ellos es la MIPSE 22 por leucemias y el otro es la MIPSE 26 por anomalías congénitas cardiovasculares. Para ambos casos su diagnóstico certero y su eventual tratamiento, siempre temprano para obtener el pronóstico más favorable posible, requieren unidades de servicio altamente especializadas y ese cuarto nivel de atención sanitaria solamente se ofrece en Costa Rica en el Hospital Nacional de Niños. Por lo tanto, normalmente cuando en las Unidades MaternoInfantiles distribuidas por todo el país, ya sean públicas o privadas, se sospecha de alguno de estos diagnósticos, se derivan los casos al mencionado hospital ${ }^{52}$.

Los problemas congénitos cardiovasculares dependen en gran medida del tiempo en horas que transcurre entre el diagnóstico y la cirugía terapéutica correctiva ${ }^{53}$. Es funda- mental para algunas patologías concretas de este grupo de MIPSE 26 disminuir dicho período. Por esa razón, en cierta medida se podrían ver perjudicados los neonatos de zonas alejadas de la capital. No obstante, aparentemente en Costa Rica se ha podido lidiar con ese problema clásico de acceso al servicio ya que afecta tanto a los cantones pobres como a la misma capital ${ }^{54}$ persistiendo algunos problemas de registro ${ }^{55,56}$.

Sí se conoce que en el Hospital Nacional de Niños existen potentes unidades de tratamiento para ambos grupos de patología, se puede descartar que en el país estos males no se atienden, por lo que la igualdad de trato a la población enferma por estas causas solamente se puede atribuir a un Sistema de Salud que ha sabido eliminar muchas barreras o limitaciones de acceso para ser tratados por estas causas.

En esta investigación se han documentado en Costa Rica desigualdades en la mortalidad innecesariamente prematura y sanitariamente evitable durante el período 20002005. Dichas desigualdades perjudican en algunas ocasiones a los cantones ricos y en otras a los cantones pobres. Finalmente, esta revisión confirma las 5 causas MIPSE más importantes de Costa Rica, enfermedad isquémica de corazón, accidentes de tránsito, accidentes cerebrovasculares, causas perinatales y suicidios.

En futuras investigaciones se pueden estudiar períodos de tiempo más amplios, discriminar los grupos MIPSE por sexo, edad, etnia, profesión, etc., con la intención de identificar desigualdades y poder anularlas. Reforzar los sistemas de registro de información oficial en el futuro será determinante para depurar las conclusiones en estudios como este.

\section{AGRADECIMIENTOS}

A Melvin Morera Salas, economista de la salud de la Caja Costarricense de Seguro 
Social (CCSS) y del Centro Centroamericano de Población (CCP) por su colaboración. A las Fundaciones Gaspar Casal y MAPFRE Medicina, por desinteresadas becas.

\section{BIBLIOGRAFÍA}

1. Kristof N. The happiest people. New York Times 6 enero de 2010. Disponible en http://www.nytimes.com/2010/01/07/opinion/07kristof.html.

2. Davey-Smith G. Income inequality and mortality: why are they related? BMJ. 1996; 312, 7039, p. 987-8.

3. Llorca Castro F. Políticas de Salud en la Unión Europea: ¿Hacia un Sistema Europeo de Salud Pública? Rev. Adm. Sanit. 2006 Oct; 4(4):727-743.

4. Rosero-Bixby L. Spatial access to health care in Costa Rica and its equity: a GIS- based study. Soc. Sci. Med. 2004; 58: 1271-1284.

5. Vargas I, Vázquez ML, Jane E. Equidad y reformas de los sistemas de salud en Latinoamérica. Cad. Salud Pública. 2002 Jul-Ago; Vol. 18, No 4: 927937.

6. Wright J, Dugdale B, Hammond I, Jarman B, Neary $\mathrm{M}$, Newton $\mathrm{D}$, et al. Learning from death: a hospital mortality reduction programme. J R Soc Med. 2006 Jun; Vol. 99: 303-308.

7. Hsiao W, Blumenthal D. Privatization and Its Discontents- The Evolving Chinese Health Care System. N Engl J Med. 2005 Sep; 353; 11: 11651170.

8. Basu J, Mobley L. Illness severity and propensity to travel along the urban - rural continuum. Health Place. 2007 Mar; 13; 381-399.

9. Asch S, Kerr E, Keesey J, Adams J, Setodji C, Malik S, et al. Who Is at Greatest Risk for Receiving Poor - Quality Health Care? N Engl J Med. 2006 Mar; 354; 11: 1147-1156.

10. Ojikutu B, Stone V. Women, Inequality and Burden of HIV. N Engl J Med. 2005 Feb; 352; 7: 649-652.

11. Phillip K, Veenstra D, Oren E, Lee J, Sadee W. Potential Role of Pharmacogenomics in Reducing Adverse Drug Reaction. JAM A 2001 Nov; 286 (18); 2270-2279.
12. Navarro V, Muntaner C, Borrell C, Benach J, et al. Politics and health outcomes. Lancet. 2006 Sep; 368: 1033-37.

13. Bleichrodt H, Van Doorslaer E. A welfare economics foundation for health inequality measurement. J Health Econ. 2006 Feb; 25; 945-957.

14. Currie A, Shields M, Wheatley Price S. The child / family health gradient: Evidence from England. J Health Econ. 2007 Sep; 26; 213-232.

15. Bautista D, Alfonso JL, Corella D, Sainz C. Influence of Social Factor son Avoidable Mortality: A Hospital - Based Case Control Study. Public Health Rep. 2005 Jan-Feb; 120: 55-62.

16. Balia S, Jones A. M. Mortality, Lifestyle and Socioeconomic Status. J Health Econ. 2007 Mar; 27(1); $1-26$.

17. Navarro V. Inequalities are Unhealthy. Mont Rev. 2004 Jun; 56; 2: 26-30.

18. Gerdtham U, Johannesson M. A note on the effect of unemployment on mortality. J Health Econ. 2003 Dec; 22; 505-518.

19. Gardner J, Oswald A. How is mortality affected by money, marriage and stress? J Health Econ. 2004 Jul; 23; 1181-1207.

20. Lynch J, Davey-Smith G, Kaplan G, House J. Income inequality and mortality: Importance to health of individual income, psychosocial environment, or material conditions. BMJ. 2000 Apr; 320, 7243, p. $1200-4$.

21. Miller D, Paxon C. Relative income, race and mortality. J Health Econ. 2006 Mar; 26; 979-1003.

22. Ortún V, Gispert R. Exploración de la mortalidad prematura como guía de política sanitaria e indicador de calidad asistencial. Med Clín (Barc). 1988; 90: 399-403.

23. Carmona Villalobos K, Ramos Esquivel M, Sánchez Matarrita F. Pobreza, Desigualdad en el Ingreso y Polarización Social a nivel de cantones y distritos en Costa Rica [tesis doctoral]. San José (CR): Universidad de Costa Rica; 2005.

24. Casco Jarquin, AM, Mita-Alban, LC. Estudio de la discrepancia entre diagnóstico de defunción clínicos y los hallazgos de autopsia en el Hospital Calderón Guardia. AMC. 2003 Mar; 45 (1): 15-19. 
25. Banegas JR, Rodríguez Artalejo F, Graciani A, Villar F, Herruzo R. Mortality attributable to cardiovascular risk factors in Spain. Eur. J Clin. Nutr. 2003 Sep; 57(1); 18-21.

26. Wilson A, Oldenburg B, Lopez A. Targeted approaches for reducing inequities in chronic disease. Med J Australia. 2003 Sep; Vol. 179: 231-232.

27. Edwards R. The problem of tobacco smoking. BMJ. 2004 Jan; 328, p. 217-9.

28. Woodward A, Laugesen M. How many deaths are caused by second hand cigarette smoke? Tob Control. 2001 Dec; 10: 383-388.

29. Leal Mateos M, Wong MClure R, Pena N, Moraima Guevara M. Vigilancia epidemiológica para el infarto agudo al miocardio, experiencia obtenida en el Hospital Calderón Guardia. AMC. 2005 AbrJun; 47(2): 84-88.

30. Roselló Araya M, Guzmán Padilla S. Evolución de la mortalidad por enfermedad isquémica del corazón e infarto agudo del miocardio en Costa Rica, 1970-2001. Rev. Panam. Salud Pública. 2004 Nov; 16(5): 295-301.

31. Yunes J, Zubarew. Mortalidad por causas violentas en adolescentes y jóvenes: un desafío para la Región de las Américas. Rev. Bras. Epidemiol. 1999 Dic; 2(3): 102-171.

32. Poulozzi L, Ryan G, Espitia Hardeman V, Xi Y. Economic development's effect on road transport related mortality among different types of road users: Across-sectional international study. Accident Anal Preve. 2007 May; 39(3): 606-617.

33. Tapia Granados JA. La Reducción del tráfico de automóviles: una política urgente de promoción de la salud. Rev. Panam. Salud Pública. 1998 Mar; 3(3): 137-151

34. Bhutta Z, Darmstadt G, Hasan B, Haws R. Community Base Interventions for Improving Perinatal and Neonatal Health Outcomes in Developing Countries: A Review of the Evidence. Pediatrics. 2005 Feb; 115(2): 519-617.

35. Álvarez Urbina J. Morbi-mortalidad de los recién nacidos de muy bajo peso en el Hospital San Juan de Dios, San José, Costa Rica. Acta Pediátr. Costarric. $2001 ; 15(2): 46-52$.

36. Droughton J, Dip E. Avoidable deaths: predictable coverage? Ment Health Pract. 2007 Feb; 10(5): 2123.
37. Granados Bloise, D. Magnitud y Tendencia del Suicidio en los cantones de Costa Rica: 1980 - 1994. Rev. Costarric. Salud Pública. 1998 Jul; 7(12): 2335 .

38. Benatar S. Health Care Reform and the Crisis of HIV and AIDS in South Africa. N Engl J Med. 2004 Jul; 351; 1: 81-92.

39. Espinoza Aguirre A. Tendencias y Factores de Riesgo del VIH - SIDA en Costa Rica 1983 - 2001. Rev. Costarric. Salud Pública. 2006 Dic; 15(29): $35-43$.

40. Stout BD, Leon MP, Niccolai LM. Nonadherence to antiretroviral therapy in HIV-positive patients in Costa Rica. AIDS Patient Care ST. 2004 May; 18(5): 297-304.

41. Batty G, Shipley MJ, Jarrett RJ, Breeze E, Marmot MG, Davey Smith G. Obesity and overweight in relation to organ-specific cancer mortality in London (UK): findings from the original Whitehall study. Int. J Obes. 2005 Jun; 29; 1267-1274.

42. McMillan D, Sattar N, Lean M, McArdle C. Obesity and Cancer. BMJ. 2006 Nov; 333, p. 1109-11.

43. Leal Mateos M, Ortiz Barboza A. Mortalidad por Cáncer de Mama en Costa Rica 1990- 1999. Rev. Costarric. Salud Pública. 2004 Dic; 13(25):

44. Sanabria Cascante R. La gestión reguladora del Ministerio de Salud en relación con la compra deservicios de salud privados por parte de la Caja Costarricense de Seguros Social, período 1994 - 2002, a Coopesalud R.L. y ASEMECO. Rev. Cienc. Adm. Financ. Segur. Soc. 2008 Jul; 12(2): 35-45.

45. Gauri V, Cercone J, Briceño R. Separating financing from provision: evidence from 10 years of partnership with health cooperatives in Costa Rica. Health Policy Plan. 2004 Sep; 19(5): 292-301.

46. Ramirez Monge A, Gutierrez-Espeleta G, Loaiciga Vega K. Familial Breast Cancer in Costa Rica: An Initial Approach. Rev. Biol. Trop. 2004 Sep; 52(3): 531-536.

47. Fernández Morales D. Indicaciones para el uso de terapia de reemplazo hormonal oral en mujeres menopáusicas mayores de 50 años. AMC. 2007 Ene-Mar; 49(1): 26-32.

48. Freer Bustamante E. El uso adecuado de protectores solares en Costa Rica. Rev. Costarric. Cienc. Méd. 1999 Jun; 20(1-2): 103-111. 
49. Moneada Jiménez J, Meneses Montero M. Hábitos de exposición solar y conocimientos sobre el cuidado de la piel en educadores físicos mexicanos, hondureños y costarricenses. Rev. Costarric. Salud Pública. 2004 Dic; 13(25): 34-41.

50. Jiménez Martén F, Cordero Alvarado R. Mortalidad en el Paciente Alcohólico. Rev. Cienc. Soc/San José. 1997 Set; 77: 77-4.

51. Bustillos Villavicencio J, Giacomin Carmiol L. Morbimortalidad Maternofetal en Embarazos de Mujeres Mayores de 35 Años. Rev. Costarric. Cienc. Méd. 2004 Ene; 25(1-2): 59-66.

52. Monge P, Wesseling C, Rodríguez AC, Cantor K, Weiderpass E, Reutfors J, et al. Childhood leukaemia in Costa Rica, 1981-96 Paediatr. Perinat. Epidemiol. 2002 Jul; 16(3): 210-218.
53. Chang R, Klitzner T. Can Regionalization Decrease the Number of Deaths for Children Who Undergo Cardiac Surgery? A Theoretical Analysis. Pediatrics. 2002 Feb; 109(2): 171-181.

54. Benavides Lara A, Umaña Solis L. Cardiopatías Congénitas en Costa Rica: análisis de 9 años de registro. Rev. Costarri. Cardiol. 2007 Ene-Abr; 9(1): 9-14.

55. Salazar Vargas C. A propósito de cardiopatías congénitas. Rev. Costarri. Cardiol. 2007 Ene-Abr; 9(1): 3-4.

56. Arocena P, García-Prado A. Accounting for quality in the measurement of hospital performance: evidence from Costa Rica. Health Econ. 2007 Jul; 16(7): 667-685. 